\title{
WhatsApp, Viber and Telegram: which is the Best for Instant Messaging?
}

\author{
Tole Sutikno ${ }^{1}$, Lina Handayani ${ }^{2}$, Deris Stiawan ${ }^{3}$, Munawar Agus Riyadi ${ }^{4}$, Imam Much Ibnu Subroto ${ }^{5}$ \\ ${ }^{1}$ Department of Electrical Engineering, Universitas Ahmad Dahlan, Yogyakarta, Indonesia \\ ${ }^{2}$ Faculty of Public Health, Universitas Ahmad Dahlan, Yogyakarta, Indonesia \\ ${ }^{3}$ Department of Computer Engineering, Universitas Sriwijaya, Palembang, Indonesia \\ ${ }^{4}$ Department of Electrical Engineering, Universitas Diponegoro, Semarang, Indonesia \\ ${ }^{5}$ Department of Informatics Engineering, Universitas Islam Sultan Agung, Semarang, Indonesia
}

\section{Article Info}

Article history:

Received Feb 2, 2016

Revised May 13, 2016

Accepted May 26, 2016

\section{Keywords:}

Encryption

Instant Messaging

Telegram

Viber

WhatsApp

\begin{abstract}
There are many free instant messengers available now which allow people to communicate with friends with texts, phone calls, videos, by sharing files, in group or not, and to maintain contact with them even internationally. But only very few of instant messengers have gained popularity and recognition. Recent studies have shown that the most popular instant messengers are WhatsApp, Viber and Telegram. Facebook acquired WhatsApp due to its huge number of users. Viber is another messenger with many integrated features that allow for phone calls and send text messages for free, and like WhatsApp there is no subscription, while Telegram offers users a completely free open-source platform without any ads, a clean interface, and (the biggest selling point) security. So, which instant messenger is best? Currently Telegram is the most downloaded messaging apps in the Google Play Store. But at the moment WhatsApp is still the winner!
\end{abstract}

Copyright (c) 2016 Institute of Advanced Engineering and Science. All rights reserved.

\section{Corresponding Author:}

Tole Sutikno,

Department of Electrical Engineering,

Faculty of Industrial Technology, Universitas Ahmad Dahlan

UAD $3{ }^{\text {rd }}$ Campus, “Jl. Prof. Dr. Soepomo, Janturan, Yogyakarta 55164, Indonesia

Email: tole@ee.uad.ac.id

\section{INTRODUCTION}

Now that smartphone usage is on the rise, instant messaging applications have become a necessity for users. There are many free instant messengers available now with the development in technology which has shown outstanding improvements in it. These services allow people to communicate with friends with texts, phone calls, videos, shared files, in groups or not, and to maintain contact with them even internationally [1-5]. But very few have gained popularity and recognition. Recent studies have shown that the most popular instant messengers are WhatsApp, Viber and Telegram. In this paper the features of all three applications will be compared.

\subsection{WhatsApp}

Recently, WhatsApp has become the most popular messenger with more than 1 billion people as its user base, and is the first messenger app to hit this milestone [2, 6, 7]. In 2014, Facebook acquired WhatsApp because of its huge number of users [8-10]. In its initial years, it charged the user as much as USD 0.99 per year (the first 12 months was a free trial period). Later, subscription charge was completely cancelled since 18 January 2016 [11, 12]. WhatsApp provides services for text and audio messaging, free voice calls and exchanging photos or videos and even for sharing of limited types of documents. But the main advantage of 
WhatsApp is its easiness for expanding the user base. When installing it, one can virtually reach all contacts in his/her address book on the phone who have installed the same apps. And with more extensive engagement between users in user-created WhatsApp groups, it acquires a healthy share in mobile space usage [3, 8, 12].

WhatsApp clearly dominates the global instant messenger market at present. The following are the reasons why it has been chosen over Viber, Telegram or others messengers: 1) WhatsApp has become a topof-mind brand in the market of instant messengers. Many people instantly recalls WhatsApp, but not with other messengers. This is because of word-of mouth promotion by the users; 2) when using WhatsApp, people can speak to most of their friends because they are already on WhatsApp; 3) the bonus with WhatsApp is that it is ads free service, although WhatsApp is not alone in this feature: many instant messengers also provide the same service. However, the choice to provide ads-free service means that the consideration of monetization lay in other part of service or it has great resources to cover the service; and 4) another added advantages of WhatsApp over other messengers is that WhatsApp provides official web-based application that runs on Windows desktop environment. Additionally, it and can be used with the Chrome web based browser [13-16].

\subsection{Viber}

Viber is arguably the messenger with the most features in it. It has numerous options that simply superior than others; e.g. it has high quality video call. Viber provides voice calling service that can dial to any mobile number, even if the dialled number is not using Viber (with additional charge, for this case). Viber offers group chat rooms just like other messenger services, and in addition to that service, it also offer so-called public chat that allow users to communicate openly, which may suit with the type of hobby group, fans club communication, or even large communities. A number of big groups on social networks often create their channel in Viber. It offers a variety of customisable features, such as dedicated background for different chat. Instead of sending photos only, it can also perform drawings with one's finger. Viber also provides official desktop version for the apps that can be used in laptops or PCs. However, despite its rich features, it fails to attract people. It is believed that its less intuitive interface (compared with WhatsApp, for example) and its controversial decision for dominant purple colour are of several reasons why it is trailing badly $[3,4,12]$.

Viber has offered voice and video call services for a few years before WhatsApp and Telegram start to provide the similar voice and video services. Due to its earlier development, it is understandable that Viber can offer better voice quality with less noise thoughout all bandwidths, in comparison with WhatsApp and Telegram. Viber also offers two modes of voice quality: the normal and HD modes, with the latter very beneficial on high bandwidth networks. Perhaps the most unique feature is the Viber Out, which make it possible to Viber users to be in contact with people who are not on the service $[3,17,18]$.

\subsection{Telegram}

Telegram is a popular messaging service that is based on open-source platform. In addition to its completely free service without any payments, it also offers ads-free environment with a clean and fast interface. Telegram was initialised in August 2013 by the Russian-born entrepreneur Pavel Durov, but the "Russian WhatsApp" is in serious competition with the giants of the industry, WhatsApp and Viber. Telegram has gained the popularity status that it becomes the most downloaded messaging apps in the Google Play Store. Telegram has earned the similar most popular social network among free downloaded apps in more than forty countries, including Germany and the United States, beating other social network apps such as Facebook, WhatsApp, WeChat, and Kik. [12, 19-22].

Telegram is easy to sign up and use. It has many similarities to WhatsApp in terms of dealing with user ID and contact. The phone number is used for user's primary identification. Once the user installs the apps, he/she can communicate with any number in their contact list that already installs the same apps. However, Telegram has more to offer. Any user can create a username as unique ID in Telegram, thus allowing others who know this username to directly contact without having to know the phone number of the respective party. Moreover, adding other's username into Telegram's contact list will not automatically reveal the phone number. By considering that phone number is such privacy for several people, this feature is a great way to enhance the privacy protection. In addition, the archive of communication is stored in cloud, thus the users do not have to worry that they lost their chats after changing phone [12, 19].

Among its benefits is the sticker shop. It is a collection of funny images that can be used in chats besides text. It can share documents in more variety of filetypes that WhatsApp, as well as image and video sharing, without size limit. Telegram is a multiple platform app that can run on Android, iOS, Windows Phone, Mac and Windows OS. Moreover, Telegram account can be accessed from multiple devices, even at a given time, and messages appears simultaneously on all devices [19, 22]. 
Telegram is designed as a light app, very fast and reliable. It provides platform for chatting in groups, with far more users can be included in a group (up to 5000 participants) than what WhatsApp capable of. Finally, Telegram also provides bots: an automatic answering account that can respond to specific text command by giving preformatted answers. The integration of bots into Telegram's API offers unprecendented applications, something that is not found on WhatsApp [19, 21, 23].

\section{FEATURE COMPARISON}

As part of the social media, instant messaging tools are now frequently used. This is in line with social cognitive theory (SCT) which emphasises reciprocal determinism in the interaction between people and their environments [24]. SCT posits that human behaviour is the product of a dynamic interplay of personal, behavioural and environmental influences. A person's behaviour can be influenced by the environment and also influence the environment. It is relevant to the current situation that the popularity of WhatsApp, Viber and Telegram are also influenced by the environment. The more people use instant messaging, the more people follow it. According to Bandura's theory [25, 26], the use of WhatsApp, Viber and Telegram can be viewed as illustrations of these reciprocal determinisms, as providers must seek to influence the environmental and social factors that influence people's behaviour in using their product.

Person-behaviour relation is in context with the bi-directional interaction of one's thoughts, emotions and biological properties. For example, a person's beliefs, self-perceptions, goals and intentions greatly influence their behaviour. A bi-directional interaction also happens between the environment and personal characteristics [24-27]. The expectations of a human as well as its beliefs and cognitive competencies are greatly constructed by influx from social and physical environment [24]. The interaction between behaviour and environment set the aspects for modification from each side. The environment partly determines the development of one's behaviour [27]; in this case it happened to perform the behaviour of people in choosing WhatsApp, Viber and Telegram. Due to people having high expectations of the products they choose, providers must try to meet these expectations. They must always improve their product with many advantages, such as high technology, low prices and being user friendly.

Table 1 shows side-by-side the feature comparisons for the above free instant messaging services to give some perspective on where they stand [12, 28, 29]. WhatsApp and Viber bring detailed info when the exact status of messages, from the moment of sending to when the messages are opened by the recipient, while Telegram includes read receipts with double ticks, but lacks the other aforementioned features. Now, all instant messengers focus on security issues, with completely private, safe and self-destructing messages. All instant messaging providers claim that end-to-end encryption is implemented. As a natural result, people have wondered which service is safer: WhatsApp, Viber or Telegram?

\section{END-TO-END ENCRYPTION}

Encryption is essential in communication system. In secure end-to-end communication, it is required that only the communicating parties can understand the messages. Eavesdropper may not read the cryptic communication, and they have to posses the cryptographic keys in order to dechiper the conversation. In messaging service, the end-to-end encryption is highly needed so that telco or internet providers and the company that runs the service cannot read the message [30]. Surveillance and tapping are not possible because the cryptographic keys are not shared for decrypting the data being communicated or stored. Therefore, any provider of end-to-end encryption cannot handover data of their customers' communication to the authorities [31]. In its early years, instant messaging applications could not promise of keeping the user's privacy. The user's messages can be stored and recorded to gain profiles of specific users. Eventually, illegal access to social media accounts may access these records. Zero-Interaction PRivacy (ZIPR) [32] and BabelCrypt [33] were the first systems introduced to address this problem that automatically retrofitting arbitrary mobile chat applications with end-to-end encryption.

Recent changes in app security have given birth to an fierce competition in offering users secure instant messaging [19], as shown in Table 2, and this has been the tendency now for many years. WhatsApp did not really have a great rep when it came to security, but things have changed now, with the latest version of the app bringing end-to end encryption [34]. On April 5, 2016, the topic was brought to people's attention again when WhatsApp, in collaboration with Open Whisper Systems, implemented end-to-end encryption. The app encrypts all the chats and data like voice messages, media, etc. end-to-end, which means that not even WhatsApp can read our messages [12, 16, 35]. Following WhatsApp's move to adding end-to-end encryption to its platform, on April 19, 2016 Viber implemented end-to-end encryption for all messages and 
Table 1. Whatsapp, Viber and Telegram specs comparison

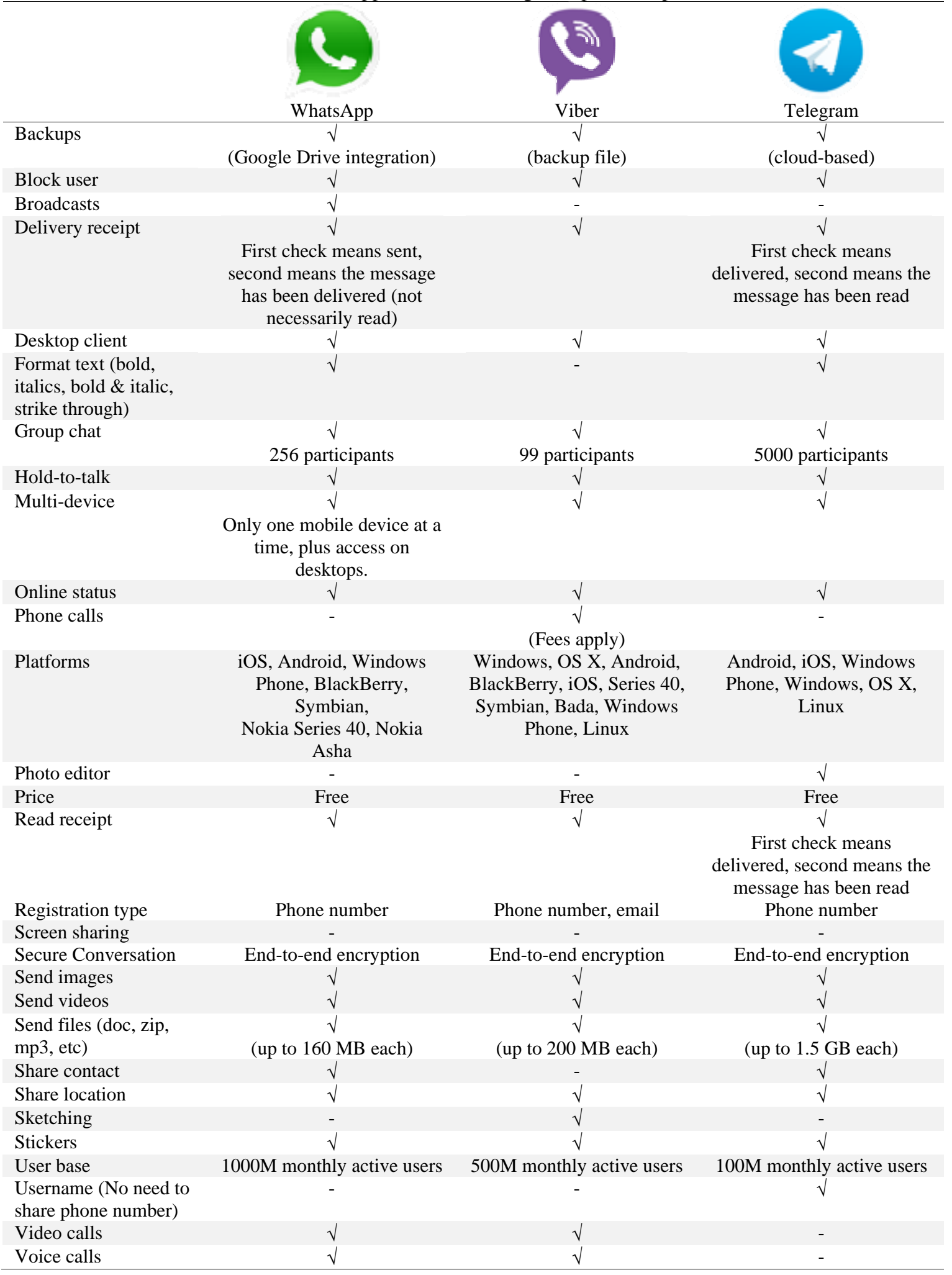

calls on its platform, including group chats (up to 200 people). Viber's end-to-end encryption is to protect its customers' communications against snooping, but only for one-to-one and group conversations in which all participants are using the latest Viber version for Android, iOS, Desktop or Windows 10 [3, 17, 18, 28]. Telegram, on the other hand, is based on the MTProto Mobile protocol and brings end-to-end encryption with secret chats. It also has the self-destruct feature and notifications to users when a screenshot is taken by the 
recipient. Moreover, Telegram is open-source, so anyone can check the app’s source code, API or protocols to find any security flaws [19-22].

Table 2. Whatsapp, Viber and Telegram in assessing the security

\begin{tabular}{|c|c|c|c|c|}
\hline Security Criteria & WhatsApp & Viber & Telegram & $\begin{array}{c}\text { Telegram } \\
\text { (Secret Chats) }\end{array}$ \\
\hline $\begin{array}{l}\text { Is communication encrypted in transit (all user } \\
\text { communications are encrypted along all the links in the } \\
\text { communication path)? }\end{array}$ & $\sqrt{ }$ & $\sqrt{ }$ & $\sqrt{ }$ & $\sqrt{ }$ \\
\hline $\begin{array}{l}\text { Is communication encrypted with a key the provider } \\
\text { does not have access to (all user communications are } \\
\text { end-to-end encrypted)? }\end{array}$ & $\sqrt{ }$ & - & $\sqrt{ }$ & $\sqrt{ }$ \\
\hline Can correspondent's identity be independently verified? & $\sqrt{ }$ & - & - & $\sqrt{ }$ \\
\hline Are past communications secured if keys are stolen? & $\sqrt{ }$ & - & - & $\sqrt{ }$ \\
\hline Is the code open to independent review? & - & - & $\sqrt{ }$ & $\sqrt{ }$ \\
\hline Is the crypto design well-documented? & $\sqrt{ }$ & - & $\sqrt{ }$ & $\sqrt{ }$ \\
\hline Has there been an independent security audit? & $\sqrt{ }$ & $\sqrt{ }$ & $\sqrt{ }$ & $\sqrt{ }$ \\
\hline
\end{tabular}

Message encryption in WhatsApp is based on the Signal Protocol combining the Double Ratchet Algorithm, prekeys and a 3-DH handshake. On the other hand, voice call encryption is with the Secure Realtime Transport Protocol (SRTP), and a separate encrypted channel is dedicated for layering all client-server communications. The Signal Protocol library used by WhatsApp is open-source and published under the GPLv3 licence [36]. Viber's protocol uses the same concepts of the double ratchet protocol used in WhatsApp in collaboration with Open Whisper Systems. However, Viber's implementation was developed from scratch and does not share Signal's source code. Viber messages are encrypted with the Salsa20 encryption algorithm. Viber calls are encrypted with 256-bit Curve25519 key-pair [3, 12, 18], while Telegram uses a symmetrical encryption scheme called MTProto. The protocol was developed by Nikolai Durov and is based on 256-bit symmetric AES encryption, RSA 2048 encryption and Diffie-Hellman key exchange. Telegram supports two layers of secure encryption. The cloud chats use server-client encryption, while secret chats employ an additional layer of client-client encryption. All data of any type is encrypted in the same way [21, 22, 37].

Even though there is no general consensus when choosing an instant messaging application, in general WhatsApp, Viber and Telegram are some of the safest apps for instant messaging. Unlike WhatsApp and Viber, Telegram's secret chat messages are fully encrypted between senders and do not give user data to third party vendors. It even offers a self-destruct feature, where messages disappear after the time set and no one sees them on your device or the recipient's.

\section{CONCLUSION}

WhatsApp is the most popular among the world's users of the smartphones with about $60 \%$, followed by Viber and, in third place, Telegram. Viber is the most functional messenger, but if the main concern is the security of communication, it is wiser to opt for Telegram. Telegram offers capability of syncronization, super fast service, reliable backup and better security feature. Although WhatsApp dominates the social media space due to its simplicity and backed by giant i.e. Facebook, Telegram is essentially providing better platform than others.

\section{References}

[1] A. Buchenscheit, B. Könings, A. Neubert, F. Schaub, M. Schneider, and F. Kargl, "Privacy implications of presence sharing in mobile messaging applications," in ACM International Conference Proceeding Series, 2014, pp. 20-29.

[2] C. Sgaras, M. T. Kechadi, and N. A. Le-Khac, "Forensics acquisition and analysis of instant messaging and VoIP applications," in Lecture Notes in Computer Science (including subseries Lecture Notes in Artificial Intelligence and Lecture Notes in Bioinformatics) vol. 8915, ed, 2015, pp. 188-199.

[3] A. L. B., P. J. N., P. V. R., and S. D. Jyoti, "Whatsapp, Skype, Wickr, Viber, Twitter and Blog are Ready to Asymptote Globally from All Corners during Communications in Latest Fast Life," Research Journal of Science and Technology, 2014, vol. 6, pp. 101-116.

[4] S. K. Khatri and K. Narula, "General maturity model for social messaging application," in 2015 4th International Conference on Reliability, Infocom Technologies and Optimization: Trends and Future Directions, ICRITO 2015, 2015. 
[5] N. H. Tang and Y. C. Lee, "A comparative study on user loyalty of mobile-instant messaging services: Korea and Vietnam (Research in Progress)," in ACM International Conference Proceeding Series, 2015.

[6] N. Statt. WhatsApp has grown to 1 billion users, 2016. Available: http://www.theverge.com/2016/2/1/10889534/whats-app-1-billion-users-facebook-mark-zuckerberg

[7] M. Seufert, T. Hoßfeld, A. Schwind, V. Burger and P. Tran-Gia, "Group-based communication in WhatsApp," 2016 IFIP Networking Conference (IFIP Networking) and Workshops, Vienna, Austria, 2016, pp. 536-541.

[8] S. Vijayakumar Bharathi and A. Goswami, "A multidimensional study on the usage of facebook among college students," International Journal of Applied Engineering Research, 2014, vol. 9, pp. 9849-9862.

[9] Chandrasekhar, C.P. The next internet bust? Economic and Political Weekly, 2014, No. 12, pp. 10-11.

[10] Vijayakumar Bharathi, S., Goswami, A. A multidimensional study on the usage of facebook among college students, International Journal of Applied Engineering Research, 2014, vol. 9, pp. 9849-9862.

[11] Mensa-Bonsu, M.A.S. The old order changeth and giveth way to the new: WhatsApp debuts as a means of substituted service in Ghana. Oxford University Commonwealth Law Journal, 2015, vol.15, pp. 149-154.

[12] Battle of the messengers: WhatsApp vs Viber vs Telegram, 2016. Available: http://appleapple.top/battle-of-themessengers-whatsapp-vs-viber-vs-telegram-poll/

[13] Mathew, A.B., Madhu Kumar, S.D. Analysis of data management and query handling in social networks using NoSQL databases. International Conference on Advances in Computing, Communications and Informatics (ICACCI), 2015, pp. 800-806.

[14] B. Khatoon, K. B. Hill, and A. D. Walmsley, "Instant messaging in dental education," Journal of Dental Education, 2015, vol. 79, pp. 1471-1478.

[15] L. Y. Jin and J. Park, "Effects of platformization strategy on continuance intention of mobile messaging apps," International Journal of Mobile Communications, 2016, vol. 14, pp. 291-308.

[16] H. Bajaj and R. Jindal, "Thinking beyond WhatsApp," in 2015 International Conference on Computing for Sustainable Global Development, INDIACom, 2015, pp. 1443-1447.

[17] U. Batavia. Detailed Comparison: Viber Vs. WhatsApp Voice Calling. Available: http://www.technewstoday.com/23384-detailed-comparison-viber-vs-whatsapp-voice-calling/

[18] E. Diaz-Aviles et al. Towards real-time customer experience prediction for telecommunication operators. Proceedings of 2015 IEEE International Conference on Big Data, 2015, pp. 1063-1072.

[19] Job, J., Naresh, V., Chandrasekaran, K. A modified secure version of the Telegram protocol (MTProto). IEEE International Conference on Electronics, Computing and Communication Technologies, CONECCT 2015, art. no. 7383884

[20] Y. Temchenko. Why does Russian-made Telegram messaging app irritate its competitors?, 2016. Available: https://goo.gl/dJMIAU

[21] How Telegram Becomes the Hottest Messaging App in the World, 2016. Available: http://neurogadget.net/2015/08/30/how-telegram-becomes-the-hottest-messaging-app-in-the-world/13950

[22] R. Sari-Motlagh, S. Ebrahimi, A. Nikfallah, S. Hajebrahimi, B. Shakiba. Lifelong Learning in Practice: The Age of Discussion through Social Media, European Urology, 2016, vol. 69, pp. 1162-1163

[23] P. Sawers. Telegram pushes supergroup limit to 5,000 people and makes groups viewable to anyone in the public, 2016. Available: http://goo.gl/UuBBOS

[24] A. Bandura, Social Foundations of Thought and Action: A Social Cognitive Theory: Prentice Hall, 1986.

[25] A. Bandura and N. E. Adams, "Analysis of self-efficacy theory of behavioral change," Cognitive Therapy and Research, 1977, vol. 1, pp. 287-310.

[26] A. Bandura, "Self-efficacy: Toward a Unifying Theory of Behavioral Change," Psychological Review, 1977, vol. 84, pp. 191-215.

[27] A. Bandura, "Human agency in social cognitive theory," American Psychologist, 1989, vol. 44, pp. 1175-1184.

[28] K. Azza. WhatsApp Vs Telegram Vs Viber, which is best for chatting?, 2016. Available: http://goo.gl/OrlqSR

[29] J. Vilches. Mobile Messaging Clients Compared, 2016. Available: http://goo.gl/Gfe9aD

[30] Hacker Lexicon: What is End-to-End Encryption?, 2016. Available: https://goo.gl/o3ZKuf

[31] J. McLaughlin. Democratic Debate Spawns Fantasy Talk on Encryption, 2016. Available: https://goo.gl/MYt1FJ

[32] A. Razeen and L. Cox, "Demo: Zero interaction private messaging with ZIPR," in MobiSys 2014 - Proceedings of the 12th Annual International Conference on Mobile Systems, Applications, and Services, 2014, pp. 351-352.

[33] A. T. Ozcan, C. Gemicioglu, K. Onarlioglu, M. Weissbacher, C. Mulliner, W. Robertson, et al., "BabelCrypt: The Universal Encryption Layer for Mobile Messaging Applications," in Financial Cryptography and Data Security: 19th International Conference, FC 2015, San Juan, Puerto Rico, January 26-30, 2015, Revised Selected Papers, R. Böhme and T. Okamoto, Eds., ed Berlin, Heidelberg: Springer Berlin Heidelberg, 2015, pp. 355-369.

[34] Tech. Telegram Vs. WhatsApp: Which Messenger to Use?, 2016. Available: http://goo.gl/8qUMiB

[35] W. S. Team. Whatsapp: End-to-End Encryption, 2016. Available: https://goo.gl/XKBtWw

[36] WhatsApp. WhatsApp Encryption Overview. Technical white paper, 2016. Available: https://www.whatsapp.com/security/WhatsApp-Security-Whitepaper.pdf

[37] MTProto Mobile Protocol, 2016. Available: https://core.telegram.org/mtproto 